\title{
Factors associated with influenza vaccine uptake during a universal vaccination programme of preschool children in England and Wales: a cohort study
}

\author{
Pia Hardelid, ${ }^{1,2}$ Greta Rait, ${ }^{3}$ Ruth Gilbert, ${ }^{1}$ Irene Petersen ${ }^{2,4}$
}

\begin{abstract}
- Additional material is published online only. To view please visit the journal online (http://dx.doi.org/10.1136/jech2015-207014).

${ }^{1}$ Population, Policy and Practice Programme, UCL Institute of Child Health, London, UK ${ }^{2}$ Research Department of Primary Care and Population Health, University College London, London, UK ${ }^{3}$ PRIMENT Clinical Trials Unit, Research Department of Primary Care and Population Health, University College London, London, UK

${ }^{4}$ Department of Clinical Epidemiology, Aarhus University, Aarhus, Denmark
\end{abstract}

\section{Correspondence to} Dr Pia Hardelid, Population, Policy and Practice Programme UCL Institute of Child Health, 30 Guilford Street, London

WC1N 1EH, UK;

p.hardelid@ucl.ac.uk

Received 25 November 2015 Revised 14 April 2016 Accepted 20 April 2016 Published Online First 13 May 2016

\begin{abstract}
Introduction Influenza vaccination through primary care has been recommended for all preschool children in the UK since 2013 as part of a universal immunisation programme. Vaccination is required annually and effectiveness varies by season. Factors associated with influenza vaccine receipt and those for other childhood vaccines may therefore differ.

Methods We used The Health Improvement Network, a large primary care database, to create a cohort of children in England and Wales aged 2-4 years eligible for vaccination in the 2014/2015 season. Mixed-effects Poisson regression models were used to determine sociodemographic and clinical factors associated with influenza vaccine receipt, allowing for practice-level variation.
\end{abstract}

Results Overall, $38.7 \%$ (95\% Cl $38.3 \%$ to $39.1 \%$ ) of 57545 children were vaccinated against influenza. Children in the poorest deprivation quintile were 19\% less likely to receive influenza vaccine than those in the wealthiest quintile (adjusted risk ratio (ARR) 0.81, 95\% $\mathrm{Cl} 0.77$ to 0.86). Children who received a timely first dose of measles-mumps-rubella vaccine were twice as likely to receive influenza vaccine (ARR $2.0095 \% \mathrm{Cl}$ 1.87 to 2.13 ). Being 4 years old, not in a clinical risk group, or living with 2 or more other children were also significantly associated with a lower probability of vaccination.

Discussion Children living in areas of higher deprivation and in larger families are less likely to receive influenza vaccine. Further research is required into whether interventions, such as offering vaccinations in other settings, could increase uptake in children, particularly in deprived areas.

\section{INTRODUCTION}

Influenza is a common infection in children, which can lead to hospital admission ${ }^{1}$ and, rarely, death. ${ }^{2}$ The benefits of vaccination for individual children include a reduced risk of confirmed influenza infection. ${ }^{4}$ These benefits are likely to be higher in children with chronic conditions, including neurological or chronic respiratory conditions, who are at increased risk of influenza-related complications, ${ }^{5}$ although there are few clinical trials of influenza vaccine in these groups.

The UK introduced a universal influenza vaccination programme for children in September 2013. This replaced a policy of selective vaccination for children at increased risk of influenza complications due to chronic conditions. ${ }^{6}$ Under the new programme, which is being progressively rolled out, preschool children (aged between 2 and 4 years inclusive) are being offered vaccine through their general practitioner (family physician). School-age children up to age 16 years will be offered vaccination at school. The live attenuated vaccine, offered to the vast majority of children under the universal programme, has a good safety profile. $^{7}$

There is a substantial body of research into determinants of routine childhood vaccines in the UK. These studies showed an inverse J-shaped relationship between deprivation indicators and the likelihood of being fully vaccinated in the first year of life. ${ }^{8} 9$ Higher parity is associated with lower uptake of childhood vaccinations, but the association between ethnic group and vaccination uptake in children is not consistent across studies. ${ }^{10-12}$

The universal influenza vaccination programme is different to other childhood immunisation programmes. For example, vaccination is required annually, and the effectiveness of vaccination varies according to the degree of match between the circulating and vaccination strains of influenza. ${ }^{3}$ Most importantly, the primary purpose of introducing the universal childhood programme was to reduce influenza transmission, and its cost-effectiveness rests on the indirect impact on the reduction of severe illness and mortality among the elderly. ${ }^{13}$ The modelling study on which the recommendation to extend influenza vaccination to all children was based showed that a universal policy would be cost-effective even at 30\% uptake in children. ${ }^{14}$

The need for high uptake is therefore less urgent for policymakers, compared with, for example, the need to ensure high uptake of the measles-mumpsrubella (MMR) vaccine. Indeed, published estimates of influenza vaccination uptake based on aggregated extracts from primary care records show that $38 \%$ and $37 \%$ of preschool children in England and Wales, respectively, were vaccinated in the $2014 / 2015$ season. ${ }^{15}{ }^{16}$ Vaccination uptake is also low $(<10 \%$ in children overall) in some jurisdictions, such as Ontario (Canada) and Western Australia, where universal influenza vaccination of children has been recommended for several years. ${ }^{17} 18$

Owing to the requirement for annual vaccination, the recent change from a selective to universal programme, and the varying effectiveness between seasons, determinants of vaccination may differ between influenza and other childhood 
vaccines. An ecological study of pilot sites for the universal childhood programme in England in 2013/2014 showed areas with higher deprivation, non-white or Muslim population to have lower uptake. ${ }^{19}$ However, it is not certain whether these effects also operate at the individual level. Large general practice databases provide individual-level information on important determinants of vaccination uptake including clinical risk factors, family structure and vaccination history. We examine child and family risk factors associated with influenza vaccination uptake in preschool children in primary care in England and Wales, and examine variation in vaccination uptake by general practice.

\section{METHODS}

\section{Data source}

We used The Health Improvement Network (THIN) for this study. ${ }^{20}$ THIN is an electronic primary care database, covering approximately $6 \%$ of the UK population registered in a primary care practice who have agreed to submit data anonymously to THIN. THIN has been found to be representative of the UK population in terms of demographic characteristics and consultation patters. ${ }^{21} 22$ THIN contains information on patient demographics, diagnoses, prescriptions, vaccinations and tests carried out in primary care; it does not cover procedures and diagnoses made in secondary care. Data are entered into patient electronic records by general practitioners (GPs, primary care clinicians) or nurses during patient consultations. Diagnoses are coded using Read codes, ${ }^{23}$ and prescriptions using drug codes, which map onto the British National Formulary. ${ }^{24}$

All THIN data are anonymised and originally collected for the purposes of clinical management. No ethnical review was sought based on advice from the NHS Health Research Authority. ${ }^{25}$ The study was reviewed and approved by the Scientific Review Committee of the data providers, IMS Health (reference number SRC 14-004).

\section{Inclusion criteria}

We examined vaccination uptake in the 2014/2015 season. This was the first season when all preschool children aged 2 years and older were offered vaccination in primary care. We extracted information from THIN on all children with complete data on the variables of interest, permanently registered with a GP practice contributing data to THIN in England or Wales, who were eligible to receive vaccination in primary care during the 2014/2015 season. To be eligible, children had to be aged between 2 and 4 years, inclusive, on the 31 August 2014. ${ }^{15}$ Vaccination status was determined on 31 January 2015 to be able to compare uptake with published figures from England (the most populous country). In order to allow a sufficient time period to define risk factors for non-vaccination, children had to be registered with a THIN practice meeting quality criteria $^{2627}$ since before their first birthday.

\section{Variable definitions}

Children who are not in risk groups (see below) are recommended to receive one dose of live attenuated influenza vaccine (LAIV), and children in risk groups are recommended to receive either one or two doses of LAIV, or inactivated vaccine (IV) depending on their condition and vaccination history. ${ }^{6}$ We determined for each child receipt of vaccination with LAIV or IV in the period between 1 September 2014 and 31 January 2015. Children who had received at least one dose of LAIV or IV during the 2014/2015 season were defined as vaccinated. Age (in single years) was calculated on 31 August 2014 based on the child's month and year of birth. Socioeconomic status is available in THIN as quintile of the Townsend score, a small-area measure of deprivation derived at Census output area-level (approximately 150 households), based on the patient postcode. ${ }^{28}$ The indicators used to derive the Townsend score (unemployment, home and car ownership and overcrowding) came from the 2001 Census. We also determined the country of residence (England or Wales). An indicator of rurality was also available based on data from the 2001 Census and linked to the patient postcode.

All patients in THIN are allocated a family number derived from the first line of the address of the patient, or other family members if registered in the same practice. We identified the number of other children (aged $<18$ years) in each family using the family number. We set the family number to 'missing' if more than six children had the same family number, to avoid misclassification into families of some children living at addresses incorporating multiple households. We selected a random child from each family within the age group eligible to receive LAIV during 2014/2015 season for inclusion in the cohort.

We used a prespecified list of Read and drug codes to identify children in clinical risk groups, ${ }^{29}$ used by the UK Department of Health to monitor vaccination uptake. It includes diagnostic codes for cystic fibrosis, bronchopulmonary dysplasia, congenital heart disease and cerebral palsy. Asthma was indicated by at least one prescription for oral steroids in a child with an asthma diagnosis code, at least one inhaled steroid prescription, or a diagnostic code indicating asthma-related hospital admission or medication. Children were classified as being in a clinical risk group if any of the relevant Read or drug codes were recorded in THIN between 31 January 2014 and 31 January 2015.

In the UK, children are recommended to receive their first dose of MMR vaccine at 12-13 months. We used timely receipt of MMR vaccine as an indicator of adherence with the childhood vaccination programme. Timely receipt of MMR vaccine was defined as at least one dose of MMR recorded between 12 and 18 months of age.

\section{Statistical analysis}

We estimated the proportion of children vaccinated against influenza (eg, received at least one dose of LAIV or IV) according to each exposure variable with 95\% CIs. Children in risk groups with no history of being immunised against influenza are recommended to receive two doses of vaccine. ${ }^{6}$ We therefore determined the proportion of at-risk children not previously vaccinated against influenza prior to the 2014/2015 season, who received two doses of vaccine according to recommendations. These children were defined as being vaccinated in the main analyses if they had received at least one dose of vaccine.

We used mixed-effects Poisson regression models to model the association between each of the exposure variables of interest and the outcome (receipt of influenza vaccination) using Stata version 13 (StataCorp, College Station, Texas, USA). College Station, Texas, USA: StataCorp LP, 2013. Unadjusted and adjusted risk ratios (ARRs) were estimated using these models. We chose to fit a Poisson regression model to be able to estimate risk ratios rather than odds ratios, since odds ratios will overestimate differences in risk when outcomes are common. ${ }^{30}$ Poisson regression models can, instead, be used to obtain estimates of the risk ratio. ${ }^{31}$ GP practice was included as a random effect to allow for practice-level variation in uptake. All exposure variables (age, Townsend quintile, number of other children in the family, presence of a chronic condition, timely receipt of 
MMR vaccination, sex, rurality and country) were considered for inclusion in the regression models, and were included in a forward stepwise procedure. Models were compared using the Akaike Information Criterion (AIC). If inclusion of an exposure variable reduced the AIC (compared to a model excluding the variable), we considered this variable to significantly improve model fit, and the variable was included in the final multivariable model. As a sensitivity analysis, we also determined whether including the full THIN record (rather than 1 year prior to 31 January 2015) made a difference to the proportion of children in a risk group, vaccine uptake in risk groups, and the ARRs by refitting the final model with this alternative method of identifying children in clinical risk groups.

To examine practice-level variation in uptake over and above that explained by variation in the variables significantly associated with uptake in the final model, we calculated adjusted vaccination uptake rates for each practice. First, we refitted the final model as a logistic regression model (without a random effect) to be able to estimate the probability of receiving influenza vaccination for each child. We then summed these probabilities by GP practice to obtain the expected number of children vaccinated. We calculated adjusted vaccination uptake for practice $j$ as

$$
\text { uptake_ad } j_{j}=\text { uptake_tot } \times \frac{\mathrm{obs}_{\mathrm{j}}}{\exp _{\mathrm{j}}}
$$

Where obs $s_{j}$ and $\exp _{\mathrm{j}}$ are the observed and expected number of children vaccinated in practice $j$, respectively, and uptake_tot is the overall uptake of influenza vaccine in the child cohort. We determined outliers using funnel plots with $95 \%$ limits calculated using normal approximation and adjusted for multiplicative overdispersion. ${ }^{32}$

\section{RESULTS}

The final sample included 57545 children, aged between 2 and 4 years on 31 August 2014 who were registered with one of 290 general practices on 31 December 2015. Exclusions, and the representativeness of the final sample, are described in online supplementary figure $\mathrm{S} 1$ and text $\mathrm{S} 1$.

Overall, 22267 (38.7\%, 95\% CI 38.3\% to 39.1\%) children had received at least one dose of influenza vaccine. The number and percentage of children who had received at least one dose of influenza vaccine according to the exposure variables are shown in table 1 . Only 3183 children (5.5\%) were classified as being in a clinical risk group using the definition used to monitor vaccination uptake by the Department of Health. The most common risk condition was asthma: 3075 of 3183 children in a risk group had asthma (96.6\%); 4428 children had received neither a timely first dose of MMR vaccine nor influenza vaccine $(7.7 \%)$.

Of the 22291 children who had received influenza vaccine, 21984 (98.6\%) received one dose, and 307 children received two doses (1.4\%); $92.7 \%$ of children who received one dose received LAIV (20 383 children). Of the 307 children who received two doses, 255 children $(83.1 \%)$ received two doses of LAIV, $40(13.0 \%)$ received two doses of IV; the remaining 12 (3.9\%) children received one dose each of LAIV and IV. There were 1782 children in clinical risk groups who had no record of receiving influenza vaccine prior to the 2014/2015 season. Of these children, $78(4.4 \%)$ had received two doses of vaccine as recommended, 618 (34.7\%) received one dose and 1086 (60.9\%) were not vaccinated at all in the 2014/2015 season.
Table 1 The number of children vaccinated and the percentage of children who received at least one dose of influenza vaccine (vaccinated) according to sociodemographic and clinical characteristics, 2014/2015 ( $n=57545)$

\begin{tabular}{|c|c|c|c|}
\hline Variable & $\begin{array}{l}\text { Number of } \\
\text { children (\%) }\end{array}$ & $\begin{array}{l}\text { Number } \\
\text { vaccinated }\end{array}$ & $\begin{array}{l}\% \text { vaccinated } \\
(95 \% \mathrm{Cl})\end{array}$ \\
\hline \multicolumn{4}{|l|}{ Age (years) } \\
\hline 2 & $20413(35.5)$ & 8315 & 40.7 (40.1 to 41.4$)$ \\
\hline 3 & $19537(34.0)$ & 8325 & 42.6 (41.9 to 43.3 ) \\
\hline 4 & 17595 (30.6) & 5651 & 32.1 (31.4 to 32.8 ) \\
\hline \multicolumn{4}{|l|}{ In clinical risk group } \\
\hline No & $54362(94.5)$ & 20604 & 37.9 (37.5 to 38.3$)$ \\
\hline Yes & $3183(5.5)$ & 1687 & 53.0 (51.2 to 54.7$)$ \\
\hline \multicolumn{4}{|c|}{ Number of other children in household } \\
\hline 0 & $17768(30.9)$ & 7164 & 40.3 (39.6 to 41.0$)$ \\
\hline 1 & 25109 (43.6) & 10259 & 40.9 (40.2 to 41.5$)$ \\
\hline 2 & $9932(17.3)$ & 3483 & 35.1 (34.1 to 36.0 ) \\
\hline 3 & $3453(6.0)$ & 1059 & 30.7 (29.1 to 32.2) \\
\hline 4 & $994(1.7)$ & 258 & 26.0 (23.3 to 28.8 ) \\
\hline 5 & $289(0.5)$ & 68 & 23.5 (18.8 to 28.9$)$ \\
\hline \multicolumn{4}{|l|}{ Townsend quintile } \\
\hline 1st (least deprived) & $13110(22.8)$ & 6113 & 46.6 (45.8 to 47.5$)$ \\
\hline 2nd & $11474(19.9)$ & 4803 & 41.9 (41.0 to 42.8$)$ \\
\hline $3 r d$ & $13026(22.6)$ & 4882 & 37.5 (36.6 to 38.3 ) \\
\hline 4th & $12178(21.2)$ & 4124 & 33.9 (33.0 to 34.7 ) \\
\hline 5th (most deprived) & $7757(13.5)$ & 2369 & 30.5 (29.5 to 31.6) \\
\hline \multicolumn{4}{|c|}{ Timely first dose MMR vaccine } \\
\hline No & $5445(9.5)$ & 1017 & 18.7 (17.7 to 19.7$)$ \\
\hline Yes & $52100(90.5)$ & 21274 & 40.8 (40.4 to 41.3$)$ \\
\hline \multicolumn{4}{|l|}{ Country } \\
\hline England & 49855 (86.6) & 19419 & 39.0 (38.5 to 39.4$)$ \\
\hline Wales & 7690 (13.4) & 2872 & 37.3 (36.3 to 38.4 ) \\
\hline \multicolumn{4}{|l|}{ Sex } \\
\hline Male & $29531(51.3)$ & 11520 & 39.0 (38.5 to 39.6$)$ \\
\hline Female & $28014(48.7)$ & 10771 & 38.4 (37.9 to 39.0$)$ \\
\hline \multicolumn{4}{|l|}{ Rurality } \\
\hline Urban & $49862(86.7)$ & 18823 & 37.8 (37.3 to 38.2 ) \\
\hline Town/fringe & $5138(8.9)$ & 2333 & 45.4 (44.0 to 46.8 ) \\
\hline $\begin{array}{l}\text { Village/hamlet/ } \\
\text { isolated dwelling }\end{array}$ & $2545(4.4)$ & 1135 & 44.6 (42.7 to 46.6$)$ \\
\hline Total & $57545(100)$ & 22291 & 38.7 (38.3 to 39.1 ) \\
\hline \multicolumn{4}{|c|}{ Type of clinical risk group } \\
\hline $\begin{array}{l}\text { Asthma or chronic } \\
\text { lung condition }\end{array}$ & $3079(5.4)$ & 1638 & $53.2(51.4$ to 55.0$)$ \\
\hline $\begin{array}{l}\text { Chronic neurological } \\
\text { condition }\end{array}$ & $22(0.04)$ & 12 & 54.5 (32.2 to 75.6$)$ \\
\hline $\begin{array}{l}\text { Chronic heart } \\
\text { condition }\end{array}$ & $35(0.06)$ & 15 & 42.9 (26.3 to 60.6$)$ \\
\hline $\begin{array}{l}\text { Diabetes/chronic } \\
\text { kidney/liver condition }\end{array}$ & $20(0.03)$ & 12 & 60.0 (36.1 to 80.9$)$ \\
\hline $\begin{array}{l}\text { Immunosuppression } \\
\text { (including spleen } \\
\text { conditions) }\end{array}$ & $28(0.05)$ & 11 & 39.3 (21.5 to 59.4$)$ \\
\hline
\end{tabular}

Townsend quintile, age, number of other children in the household, timely receipt of MMR vaccine, and being in a clinical risk group were independently associated with receiving at least one dose of influenza vaccine in the final Poisson regression model (table 2). The unadjusted and adjusted risk ratios were very similar, indicating that there was minimal confounding between the explanatory variables. The strongest association was seen for timely receipt of MMR vaccine; with children 
Table 2 Unadjusted risk ratios and adjusted risk ratios from mixed effects Poisson regression models (allowing for practice-level clustering) by the key risk factors ( $n=57545)^{*}$

\begin{tabular}{|c|c|c|}
\hline Variable & $\begin{array}{l}\text { Unadjusted risk } \\
\text { ratio }\end{array}$ & $\begin{array}{l}\text { Adjusted risk ratio } \\
\text { (ARR) } \dagger\end{array}$ \\
\hline \multicolumn{3}{|l|}{ Age (years) } \\
\hline 2 & 1 & 1 \\
\hline 3 & 1.04 (1.01 to 1.08$)$ & 1.04 (1.01 to 1.07$)$ \\
\hline 4 & 0.78 (0.76 to 0.81$)$ & 0.79 (0.76 to 0.82$)$ \\
\hline \multicolumn{3}{|l|}{ In clinical risk group } \\
\hline No & 1 & 1 \\
\hline Yes & 1.44 (1.37 to 1.51$)$ & 1.45 (1.38 to 1.53$)$ \\
\hline \multicolumn{3}{|c|}{ Number of other children in household } \\
\hline 0 & 1 & 1 \\
\hline 1 & 0.98 (0.95 to 1.01$)$ & 0.99 (0.96 to 1.02$)$ \\
\hline 2 & $0.86(0.82$ to 0.89$)$ & 0.90 (0.86 to 0.94$)$ \\
\hline 3 & 0.77 (0.72 to 0.82$)$ & 0.82 (0.77 to 0.88 ) \\
\hline 4 & 0.67 (0.59 to 0.76$)$ & 0.74 (0.65 to 0.84$)$ \\
\hline 5 & 0.60 (0.47 to 0.76$)$ & $0.68(0.53$ to 0.86$)$ \\
\hline \multicolumn{3}{|l|}{ Townsend quintile } \\
\hline 1st (least deprived) & 1 & 1 \\
\hline 2nd & 0.95 (0.91 to 0.98 ) & 0.95 (0.91 to 0.98$)$ \\
\hline $3 r d$ & 0.90 (0.86 to 0.93$)$ & 0.90 (0.87 to 0.94$)$ \\
\hline 4th & 0.84 (0.81 to 0.88$)$ & $0.86(0.82$ to 0.89$)$ \\
\hline 5th (most deprived) & $0.79(0.75$ to 0.83$)$ & 0.81 (0.77 to 0.86$)$ \\
\hline \multicolumn{3}{|c|}{ Timely first dose MMR vaccine } \\
\hline No & 1 & 1 \\
\hline Yes & 2.08 (1.95 to 2.22$)$ & 2.00 (1.87 to 2.13$)$ \\
\hline \multicolumn{3}{|l|}{ Country* } \\
\hline England & 1 & - \\
\hline Wales & 1.02 (0.88 to 1.17$)$ & \\
\hline \multicolumn{3}{|l|}{ Sex* } \\
\hline Male & 1 & - \\
\hline Female & 0.99 (0.96 to 1.01$)$ & \\
\hline \multicolumn{3}{|l|}{ Rurality* } \\
\hline Urban & 1 & - \\
\hline Town/fringe & 1.05 (0.98 to 1.13$)$ & \\
\hline $\begin{array}{l}\text { Village/hamlet/isolated } \\
\text { dwelling }\end{array}$ & $1.02(0.94$ to 1.10$)$ & \\
\hline
\end{tabular}

twice as likely to receive influenza vaccine if they also received timely MMR vaccine compared to children who did not (ARR: 2.00 (95\% CI 1.87 to 2.13). Children living in the fifth most deprived areas were $19 \%$ less likely to receive influenza vaccine compared to children living in the least deprived areas (ARR $0.81,95 \%$ CI 0.77 to 0.86 ). Living with two or more other children also decreased the probability of being vaccinated: for example, children living with two other children were $10 \%$ less likely, and those living with five other children (although rare) were $32 \%$ less likely to be vaccinated than children living with no other children (ARRs $0.90,95 \%$ CI 0.86 to 0.94 and 0.68 , 95\% CI 0.53 to 0.86 respectively).

Extending the time period used to determine whether a child was in a risk group increased the number of children in risk groups from 3183 to 5258 (9.1\% of all children), 2588 $(48.5 \%)$ of whom were vaccinated. Inclusion of the revised risk group variable in the model reduced the ARR for risk group to 1.36 (95\% CI 1.30 to 1.41$)$. The relative change in all other ARRs was $<1 \%$.
Practice-level vaccination uptake varied from $0 \%$ to $88.1 \%$; the interdecile range was equal to $41.9 \%(17.4-59.3 \%)$. The substantial variation in uptake remained after adjustment for the risk factors included in the final model (figure 1).

Twenty-five practices fell outside the $95 \%$ control limits of the funnel plot, whereas only 15 practices would be expected to fall outside the limits based on random variation alone.

\section{DISCUSSION}

In this large study of over 50000 children, we found that $<40 \%$ were vaccinated against influenza under the universal programme in England and Wales. Living in a deprived area, or in a family with two or more other children were significant risk factors for not being vaccinated against influenza. By contrast, being in a clinical risk group was associated with an increased likelihood of receiving the influenza vaccine. However, only half the children at increased risk of influenza complications due to chronic conditions were vaccinated, and $<5 \%$ of children who were recommended to receive two doses of vaccine did so. We also identified substantial between-practice variation in influenza vaccination uptake.

Vaccination information in primary care is well recorded, since GPs are reimbursed by commissioners for vaccinating their patients, and the proportion of children in the cohort who were vaccinated against influenza was similar to published figures for vaccination uptake in England and Wales. ${ }^{15} 16$

The main weakness of the study is that information on some risk factors for low vaccination uptake, including ethnicity ${ }^{11}$ and maternal education ${ }^{12}$ are either sparsely recorded or not available in primary care databases. Ethnic group in particular has been associated with childhood vaccination uptake in previous studies, and area-level ethnic composition and influenza vaccination uptake were associated in a pilot of the universal influenza vaccination programme in England. ${ }^{19}$ The porcine component of LAIV makes ethnic group of particular interest for influenza vaccine. ${ }^{33}$ Differences in ethnic group composition may partially explain some of the observed associations of deprivation and number of other children in the family with vaccination In addition, inclusion of ethnic group as a variable in the regression models is likely to have improved model fit. Ethnic group recording is improving over time in UK primary care databases. ${ }^{34}$ Future studies of influenza vaccine in children using these data could, therefore, assess the effect of ethnic group on uptake.

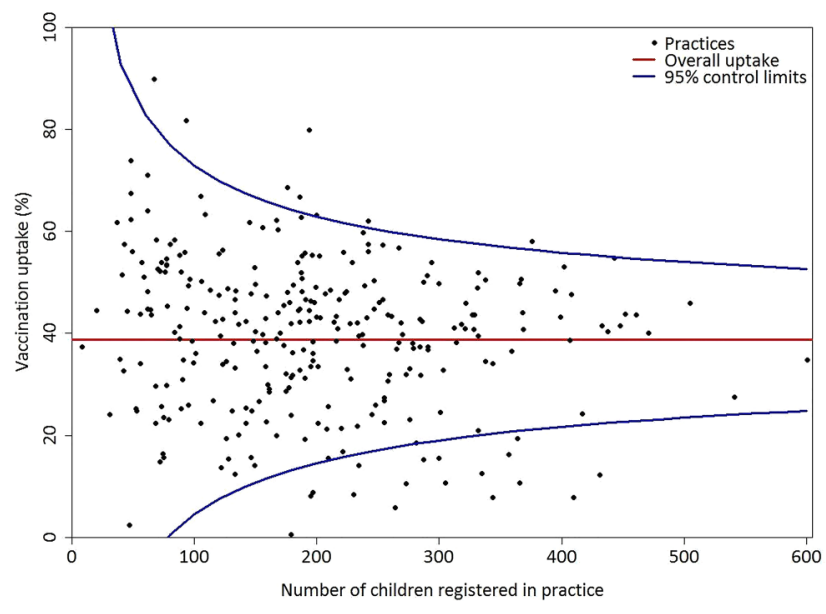

Figure 1 Funnel plot of adjusted vaccination uptake rates with 95\% overdispersion adjusted control limits. 
THIN only covers vaccination given in primary care. Children vaccinated in other settings, including hospitals or pharmacies, would be misclassified as unvaccinated. There are no national data on influenza vaccinations provided in hospitals. Vaccination in hospital is only likely to be offered to children with chronic conditions managed in secondary rather than primary care-a very small proportion of children, overall. Likewise, national data on influenza vaccinations in pharmacies are not available, but in the 2013/2014 season at least, influenza vaccinations in community pharmacies were only widely accessed in some English localities. ${ }^{35}$

We found an inverse association between area-level deprivation and influenza vaccination uptake. Studies of influenza vaccination uptake across all ages ${ }^{36}$ and in risk groups ${ }^{37}$ have shown similar results. The pilot study of the universal childhood influenza vaccination programme using deprivation determined at the GP practice level ${ }^{19}$ showed a similar difference between the wealthiest and poorest deprivation quintile (12\% cf. $16 \%$ here). Our finding of a negative association between the number of other children in the household and the likelihood of being vaccinated has also been found for MMR vaccine. ${ }^{12}$ Socioeconomic deprivation has been associated with higher risk of influenza complications including hospital admission ${ }^{1}$ and death. ${ }^{38}$ Higher parity is associated with a higher risk of respiratory hospital admissions in children. ${ }^{39}$ These findings indicate that efforts to increase vaccination uptake should be targeted to larger families and more deprived areas.

We identified wide variation in uptake by GP practice even after adjusting for the individual-level risk factors. Some of this variation is likely to be explained by differences in the prevalence of risk factors that we could not measure and, hence, adjust for, for example, ethnic group. Practice-level variation in access to vaccination for children, such as systems for inviting children to be vaccinated and opening hours, may also contribute to the observed variation. There is not a national target for influenza vaccine uptake in children, although NHS England states that an uptake rate of $40 \%$ should be achievable in the $2015 / 2016$ season, and all children should be invited to be vaccinated. ${ }^{40} \mathrm{~A}$ recent systematic review showed that reminder letters appeared to increase uptake in children in risk groups in the USA. However, there is little research into other strategies, particularly in a non-US setting. ${ }^{41}$ Interventions for improving uptake of influenza vaccine in children, particularly in risk groups, should therefore be seen as a research priority. The effect of improving access to vaccination through pharmacies, nurseries or local children's centres in deprived areas, or in secondary care for children with chronic conditions should be assessed. Integration of national data collection streams including primary care and child health records is required to measure the impact of such interventions.

Uptake in the national influenza vaccination programme is therefore substantially lower than for other routine childhood vaccinations in the UK. This could be due to several factors. Influenza is a new vaccine in the UK, and the uncertainties about effectiveness and safety may discourage parents. However, rotavirus vaccine was introduced in June 2013, and 88\% uptake was achieved for two doses by March 2015 in England. ${ }^{42}$ However, unlike the rotavirus vaccine which is given at the same time as other routine infant vaccines, influenza vaccination in preschool children requires a special appointment with the GP. Qualitative studies show that apart from difficulties in accessing vaccination clinics, parental perceptions of influenza as a less severe illness and worries about side effects all play a part in parents' decision to vaccinate, ${ }^{43}$ and such factors may contribute to low vaccination uptake. This has been compounded by uncertainties about the effectiveness of influenza vaccine, particularly during the 2014/2015 season, when there was a poor match between circulating and vaccine strains, ${ }^{44}$ which was widely reported by the media. Further studies are required to examine whether influenza vaccine can reduce the risk of hospital admissions and deaths in children, and to quantify the risk of adverse events, particularly among children in risk groups.

The influenza vaccination programme in preschool children has the lowest uptake of any of the vaccines offered through the universal childhood immunisation programme in the UK. This may be due to parental perceptions of influenza as a low-risk illness, or a lack of access to vaccination services. Strategies to increase uptake should be targeted to children at increased risk of influenza complications, such as children in risk groups, deprived areas or larger families.

\section{What is already known on this subject}

- Preschool children in the UK are offered influenza vaccine annually in primary care as part of a universal programme introduced in 2013.

- A previous study suggested areas with higher deprivation, or higher proportions of non-white or Muslim populations had lower influenza vaccine uptake in children.

- There is little data on individual-level demographic, socioeconomic or clinical predictors of influenza vaccination uptake in preschool children in the UK.

\section{What this study adds}

Only $39 \%$ of children were vaccinated overall; higher deprivation, living with two or more other children, age and not receiving a timely first dose of measles-mumps-rubella vaccine were significantly associated with not being vaccinated for influenza.

Contributors PH and IP conceived the study with GR. PH extracted and analysed the data and drafted the manuscript. All authors critically reviewed the manuscript.

Funding $\mathrm{PH}$ is funded by a National Institute for Health Research Post Doctoral Fellowship (PDF-2013-06-004). This article represents independent research funded by the National Institute for Health Research (NIHR). The views expressed are those of the authors and not those of the NHS, the NIHR or the Department of Health. RG and PH are members of the Farr Institute of Health Informatics Research London.

Competing interests None declared.

Provenance and peer review Not commissioned; externally peer reviewed.

Data sharing statement THIN data were purchased from IMS Health (previously CSD Medical Research, see: http://www.epic-uk.org/) by UCL for use in public health research. The data are under license which prevents UCL from distributing the data to third parties. The data can be obtained by contacting IMS Health by phone at +44(0) 2073888215 or email on hbhullar@uk.imshealth.com.

\section{REFERENCES}

1 Dharan NJ, Sokolow LZ, Cheng PY, et al. Child, household, and caregiver characteristics associated with hospitalization for influenza among children 6-59 months of age: an emerging infections program study. Pediatr Infect Dis J 2014;33: e141-50. 
2 Sachedina N, Donaldson LJ. Paediatric mortality related to pandemic influenza A H1N1 infection in England: an observational population-based study. Lancet 2010;376:1846-52.

3 Jefferson T, Rivetti A, Di Pietrantonj $C$, et al. Vaccines for preventing influenza in healthy children. Cochrane Database Syst Rev 2012;8:CD004879.

4 Osterholm MT, Kelley NS, Sommer A, et al. Efficacy and effectiveness of influenza vaccines: a systematic review and meta-analysis. Lancet Infect Dis 2012;12:36-44.

5 Gill PJ, Ashdown HF, Wang K, et al. Identification of children at risk of influenza-related complications in primary and ambulatory care: a systematic review and meta-analysis. Lancet Respir Med 2015:3:139-49.

6 Department of Health. Immunisation against infectious disease-The Green Book. London: The Stationery Office, 2006.

7 Belshe RB, Edwards KM, Vesikari T, et al. Live attenuated versus inactivated influenza vaccine in infants and young children. N Engl J Med 2007;356:685-96.

8 Wright JA, Polack C. Understanding variation in measles-mumps-rubella immunization coverage — a population-based study. Eur J Public Health 2006;16:137-42.

9 Samad L, Tate AR, Dezateux C, et al. Differences in risk factors for partial and no immunisation in the first year of life: prospective cohort study. BMJ 2006;332:1312-13.

10 Samad L, Butler N, Peckham C, et al. Incomplete immunisation uptake in infancy: maternal reasons. Vaccine 2006;24:6823-9.

11 Baker D, Garrow A, Shiels C. Inequalities in immunisation and breast feeding in an ethnically diverse urban area: cross-sectional study in Manchester, UK. J Epidemiol Community Health 2011;65:346-52.

12 Pearce A, Law C, Elliman D, et al. Factors associated with uptake of measles, mumps, and rubella vaccine (MMR) and use of single antigen vaccines in a contemporary UK cohort: prospective cohort study. BMJ 2008;336:754-7.

13 Department of Health. JCVI Statement on the Annual Influenza Vaccination Programme-Extension of the Programme to Children. 2012. https://www.gov.uk/ government/uploads/system/uploads/attachment_data/file/224775/

JCVI-statement-on-the-annual-influenza-vaccination-programme-25-July-2012.pdf (accessed 7 Sep 2015).

14 Baguelin M, Flasche S, Camacho A, et al. Assessing optimal target populations for influenza vaccination programmes: an evidence synthesis and modelling study. PLoS Med 2013;10:e1001527.

15 Department of Health. Influenza immunisation programme for England: GP patient groups. Data collection survey season 2014-2015. 2015. https://www.gov.uk/ government/uploads/system/uploads/attachment_data/file/429612/Seasonal_Flu_ GP Patient_Groups_Annual_Report_2014 15.pdf (accessed 7 Sep 2015).

16 Public Health Wales. Seasonal influenza in Wales-2014/15 Annual Report. 2015. http://www2.nphs.wales.nhs.uk:8080/VaccinationsImmunisationProgsDocs.nsf/(\$All)/ AECFDA367EA8E8F580257EC8002EE440/\$File/Seasonal20influenza20 vaccine20uptake20in20Wales20201415_v1a.pdf (accessed 28 Sep 2015).

17 Campitelli MA, Inoue M, Calzavara AJ, et al. Low rates of influenza immunization in young children under Ontario's Universal influenza immunization program. Pediatrics 2012;129:e1421-30.

18 Government of Western Australia Department of Health. Disease Watch: Child influenza vaccination rate low despite rise in uptake. 2013. http://www.health.wa. gov.au/diseasewatch/vol17_issue4/child_vaccination.cfm (accessed 1 Jan 2015).

19 Green HK, Andrews N, Letley L, et al. Phased introduction of a universal childhood influenza vaccination programme in England: population-level factors predicting variation in national uptake during the first year, 2013/14. Vaccine 2015;33:2620-8

20 IMS Health. The Health Improvement Network [website]. 2015. http://www.epic-uk org/our-data/statistics.shtml

21 Blak BT, Thompson M, Dattani H, et al. Generalisability of The Health Improvement Network (THIN) database: demographics, chronic disease prevalence and mortality rates. Inform Prim Care 2011;19:251-5.

22 Bourke A, Dattani H, Robinson M. Feasibility study and methodology to create a quality-evaluated database of primary care data. Inform Prim Care 2004;12:171-7.
23 Benson T. The history of the Read Codes: the inaugural James Read Memorial Lecture 2011. Inform Prim Care 2011:19:173-82.

24 Joint Formulary Committee. British National Formulary 66. London: BMJ Group, 2014.

25 NHS Health Research Authority. Does my project require review by a Research Ethics Committee? 2011. http://www.hra.nhs.uk/documents/2013/09/ does-my-project-require-rec-review.pdf

26 Horsfall L, Walters K, Petersen I. Identifying periods of acceptable computer usage in primary care research databases. Pharmacoepidemiol Drug Saf 2013;22:64-9.

27 Maguire A, Blak BT, Thompson M. The importance of defining periods of complete mortality reporting for research using automated data from primary care. Pharmacoepidemiol Drug Saf 2009;18:76-83.

28 Townsend P. Deprivation. J Soc Policy 1987;16:125-46.

29 PRIMIS. Seasonal Influenza Vaccine Uptake Reporting Specification Collection 2014/ 2015. 2014. http://www.nottingham.ac.uk/primis/documents/specs/ seasonal-flu-lqd-specification-14-15-v7.pdf (accessed 1 Oct 2015)

30 Davies HT, Crombie IK, Tavakoli M. When can odds ratios mislead? BMJ 1998;316:989-91.

31 Cummings P. Methods for estimating adjusted risk ratios. Stata J 2009;9:175-96.

32 Spiegelhalter DJ. Funnel plots for comparing institutional performance. Stat Med 2005;24:1185-202.

33 Public Health England. The children's flu vaccination programme, the nasal flu vaccine Fluenz and porcine gelatine. 2014. https://www.gov.uk/government/uploads/system/ uploads/attachment_data/file/386842/2902998_PHE_FluPorcine_QAforParents FINAL_CT.pdf (accessed 23 Nov 2015)

34 Mathur R, Grundy E, Smeeth L. Availability and use of UK based ethnicity data for health research National Centre for Research Methods Working Paper 01/13. 2013. http://eprints.ncrm.ac.uk/3040/1/Mathur-_Availability_and_use_of_UK_based_ ethnicity_data_for_health_res_1.pdf (accessed 29 Oct 2015).

35 Kassianos $G$, White $S$, Reynolds AJ, et al. Review of the experiences from the first childhood influenza vaccination programme with a live attenuated influenza vaccine in England and Scotland. Drugs Context 2015;4:212280.

36 Coupland C, Harcourt S, Vinogradova Y, et al. Inequalities in uptake of influenza vaccine by deprivation and risk group: time trends analysis. Vaccine 2007:25:7363-71.

37 Sammon CJ, McGrogan A, Snowball J, et al. Factors associated with uptake of seasonal and pandemic influenza vaccine among clinical risk groups in the UK: an analysis using the General Practice Research Database. Vaccine 2012;30: 2483-9.

38 Rutter PD, Mytton OT, Mak M, et al. Socio-economic disparities in mortality due to pandemic influenza in England. Int J Public Health 2012;57:745-50.

39 Paranjothy S, Dunstan F, Watkins WJ, et al. Gestational age, birth weight, and risk of respiratory hospital admission in childhood. Pediatrics 2013;132:e1562-9.

40 Department of Health. The national flu immunisation programme 2015/16. 2015. https://www.gov.uk/government/uploads/system/uploads/attachment_data/file/ 418428/Annual_flu_letter_24_03_15__FINALv3_para9.pdf (accessed 29 Oct 2015).

41 Aigbogun NW, Hawker JI, Stewart A. Interventions to increase influenza vaccination rates in children with high-risk conditions - a systematic review. Vaccine 2015:33:759-70.

42 Public Health England. Rotavirus infant immunisation programme 2014/15: Vaccine uptake report on the temporary sentinel data collection for England. 2015. https:// www.gov.uk/government/uploads/system/uploads/attachment_data/file/440456/ RotavirusGatewayFinalVersion.pdf (accessed 3 Mar 2016).

43 Sampson R, Wong L, Macvicar R. Parental reasons for non-uptake of influenza vaccination in young at-risk groups: a qualitative study. Br J Gen Pract 2011;61: e386-91.

44 Pebody RG, Warburton F, Ellis J, et al. Low effectiveness of seasonal influenza vaccine in preventing laboratory-confirmed influenza in primary care in the United Kingdom: 2014/15 mid-season results. Euro Surveill 2015;20:21025. 